\title{
Systematic design of slow-light photonic waveguides
}

\author{
Matzen, René; Jensen, Jakob Søndergaard; Sigmund, Ole
}

Published in:

Optical Society of America. Journal B: Optical Physics

Link to article, DOI:

10.1364/JOSAB.28.002374

Publication date:

2011

Document Version

Publisher's PDF, also known as Version of record

Link back to DTU Orbit

Citation (APA):

Matzen, R., Jensen, J. S., \& Sigmund, O. (2011). Systematic design of slow-light photonic waveguides. Optical Society of America. Journal B: Optical Physics, 28(10), 2374-2382. https://doi.org/10.1364/JOSAB.28.002374

\section{General rights}

Copyright and moral rights for the publications made accessible in the public portal are retained by the authors and/or other copyright owners and it is a condition of accessing publications that users recognise and abide by the legal requirements associated with these rights.

- Users may download and print one copy of any publication from the public portal for the purpose of private study or research.

- You may not further distribute the material or use it for any profit-making activity or commercial gain

- You may freely distribute the URL identifying the publication in the public portal

If you believe that this document breaches copyright please contact us providing details, and we will remove access to the work immediately and investigate your claim 


\title{
Systematic design of slow-light photonic waveguides
}

\author{
René Matzen,* Jakob S. Jensen, and Ole Sigmund \\ Department of Mechanical Engineering, Technical University of Denmark, Nils Koppels Allé, \\ Building 404, 2800 Kgs. Lyngby, Denmark \\ ${ }^{*}$ Corresponding author: rmat@mek.dtu.dk
}

Received May 25, 2011; revised August 15, 2011; accepted August 15, 2011; posted August 15, 2011 (Doc. ID 148149); published September 6, 2011

A pulse-delaying optimization scheme based on topology optimization for transient response of photonic crystal structures (PhCs) is formulated to obtain slow-light devices. The optimization process is started from a qualified W1 $\mathrm{PhC}$ waveguide design with group index $n_{g} \approx 40$ obtained from a simple Edisonian parameter search. Based on this, the proposed pulse delaying and subsequent pulse restoring strategies yield a design that increases the group index by $75 \%$ to $n_{g} \approx 70 \pm 10 \%$ for an operational full-width at half-maximum (FWHM) bandwidth $B_{\mathrm{FWHM}}=6 \mathrm{~nm}$, and simultaneously minimizes interface penalty losses between the access ridge and the $\mathrm{W} 1 \mathrm{PhC}$ waveguide. To retain periodicity and symmetry, the active design set is limited to the in-/outlet region and a distributed supercell, and manufacturability is further enhanced by density filtering techniques combined with material phase projections. (c) 2011 Optical Society of America

OCIS codes: $\quad 000.4430,130.5296,230.7400$.

\section{INTRODUCTION}

It is well known that the speed of light is an upper bound for waves conveying matter, energy, or information [1]. However, after the discovery of the slow-light phenomenon as a result of material dispersion, it seems that no lower limit for the group velocity at which light can travel exists [2, 3 ]. Since then, this promising technology has attracted much attention, as the application prospects are numerous, e.g., in future optical networks and information processing systems. To mention only a few remarkable properties, slow light offers the opportunity for accurate time-domain processing of optical signals, low power consumption in optical switching devices [4] , spatial pulse compression [5] , optical buffering []], and enhancement of weak linear as well as nonlinear light-matter interaction processes [7-10]. In the realization of on-chip integration of slow-light devices equipped with these features, photonic crystals (PhCs) show very promising candidacy. This is due to their intrinsic photonic bandgap (PBG) property that inhibits the existence of optical modes at certain frequency bands [11-13]. The PBG facilitates the structural engineering of PhC waveguides (-WG) [14] and microcavities (-MC) [15] that, separately or in combinations, exhibit unique tunable (groupvelocity) dispersion characteristics. In contrast to conventional semiconductors, PhCs exploit all-optical processes, thus leaving it as an obvious choice of platform for designing efficient slow-light devices [16-20]. At very low group velocities, however, this advantage is counteracted by losses that scale as the inverse square of the group velocity due to the increased density of the states [21].

Recently, inverse problem techniques have surfaced as competitive design tools to previous Edisonian approaches in the engineering of PhCs [22]. In the present paper, we will employ the methodology behind topology optimization [23] as a means to structurally design devices that slow down the speed of light. The design process is based on a time-domain analysis, and the setup consists of a PhC-WG of finite length with in- and outlet connections to ridge waveguides. The entire structure is surrounded by a perfectly matched layer (PML) as absorbing boundary conditions.

Topology optimization has previously exploited the tunability with which the PhC-WGs and -MCs are naturally born to optimize various $\mathrm{PhC}$ devices with large enhancement in optical properties. This comprises frequency-domain optimization of a $90^{\circ}$ bend [24], a T-junction [25], and a termination [26]. Other efficient $\mathrm{PhC}-\mathrm{WG}$ components have also been devised and experimentally verified in [27-30]. Even though the above examples rely on frequency-domain analyses, timedomain optimization is continuously maturing as an attractive approach. The reason hereto is its ability to handle broadband signals by short pulse excitation with a single analysis cycle, and it allows for local permutations at the frequency level. Hence, time-domain optimization facilitates the treatment of active media and nonlinearities that give rise to frequency modulation. Additionally, it can manage time-domain processing of optical signals, such as pulse shaping [31,32] and pulse delay [33], as well as optimization of PhC notch filters [34]. A comprehensive review on topology optimization of nanophotonic devices is provided in [35].

In comparison to slow-light devices based on material resonant enhancement, e.g., caused by electromagnetically induced transparency [36], the structural engineering counterpart is highly preferred because it accommodates a simpler control of light. The reason hereto is that signals very often in practice propagate in transparent media, i.e., at the operation frequency, far away from any material resonances [37]. However, both approaches are subject to the intrinsic detrimental effect that large time delays are only possible near large changes in the amplitude response (near the band edges of filter pass or stop bands) and are therefore accompanied by severe amplitude distortion. Very recently, topology optimization based on eigenvalue analyses has been used to enhance the slow-light performance of PhC-WGs by engineering/ tailoring their dispersion properties [38,39]. Both approaches include manufacturing constraints, and in particular the latter 
successfully incorporates robustness to further enhance manufacturability $[\underline{40}, \underline{41}]$.

The optimization process is, in the present paper, completed in two consecutive steps: first, a pulse delay step where the objective is the time delay of the pulse while its shape is preserved through a relaxed signal shaping constraint. Next, a pulse restoring step is carried out in which the pulse shape is tailored to match the original reference pulse in the exit ridge waveguide, but now with the maximized delay obtained in first step fixed in a pulse conforming objective function.

\section{FORMULATION}

The method presented in this paper supports propagation of transverse electric (TE) modes within two-dimensional (2D) optical waveguide structures. The medium residing inside these structures occupies a composite of regions of homogeneous dielectric material in the $x-y$ plane and is invariant in the $z$ direction.

With these assumptions, the behavior is governed by the following scalar-wave equation:

$$
\begin{aligned}
& \frac{\partial}{\partial x}\left(A \frac{\partial \Psi}{\partial x}\right)+\frac{\partial \Psi}{\partial y}\left(A \frac{\partial \Psi}{\partial y}\right)-\frac{B}{c^{2}} \frac{\partial^{2} \Psi}{\partial t^{2}}=0 \\
& \Psi=H_{z}(\mathbf{r}, t), \quad A=1 / \varepsilon_{r}(\mathbf{r}), \quad B=1,
\end{aligned}
$$

where $H_{z}$ is the transverse components of the magnetic field, as a function of position $\mathbf{r}=(x, y)$ and time $t ; c$ is the vacuum speed of light; and $\varepsilon_{r}(\mathbf{r})$ is the relative permittivity that only varies spatially. The structures are surrounded by PML regions to minimize nonphysical reflection from the boundaries. The formulation in Eq. (1) then needs to be modified to handle the anisotropic, dispersive PML behavior, which can be found in [34]. The formulation can equally be employed for transverse magnetic modes by letting $\Psi=E_{z}(\mathbf{r}, t), A=1$, and $B=\varepsilon_{r}(\mathbf{r})$, where $E_{z}$ denotes the out-of-plane electric field component.

Because we consider macroscopic systems containing dielectric material, we utilize that no fundamental length scale exists by introducing $\left(x^{\prime}, y^{\prime}\right)=(x, y) / a$, where $a$ is the lattice constant or the pitch of the PhC [42]. Based on Eq. (1), this leads to scaled time $t^{\prime}=t c / a$ and scaled angular frequency $\omega^{\prime}=\omega a / c$. We use the scaled parameters in the remainder of this paper unless otherwise stated, and it is straightforward to switch between these and the nonscaled parameters as soon as $a$ is known a priori.

To realize the slow-light device, we exploit the unique tunable dispersion properties of a W1 PhC-WG given in Fig. 1, where a single row of holes has been removed. As illustrated, the waveguide has in- and outlet connections to straight ridge waveguides, and the crystal is formed by perforated air holes with radius $r / a=0.30$ in the dielectric material GaAs with $\varepsilon=11.4$. This yields a bandgap in the normalized frequency $a / \lambda=0.21-0.30$, where $\lambda$ denotes the wavelength. We will assume that the pitch of the lattice is $a=370 \mathrm{~nm}$.

To seek a numerical solution to Eq. (1), the computational domain, including the PML regions, is divided into bilinear square elements. Then, applying the standard finite-element technique [43] yields the system of ordinary differential equations

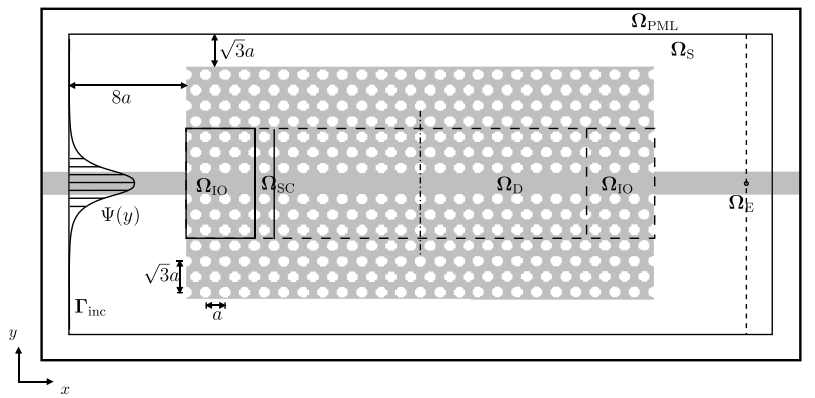

Fig. 1. Ridge waveguide optimization setup. The computational domain contains a solution region $\Omega_{\mathrm{S}}$, PML region $\Omega_{\mathrm{PML}}$, and design region $\Omega_{\mathrm{D}}$ encapsulated by the dashed boundary. The "active" design set consists of a $y$ mirrored in-/outlet region $\Omega_{\mathrm{IO}}$ and a $x-y$ mirrored supercell $\Omega_{\mathrm{SC}}$ that is a subset of $\Omega_{\mathrm{D}}$. Whenever an optimized design is presented, we only show the material distribution in $\Omega_{\mathrm{IO}}$ and $\Omega_{\mathrm{SC}}$. The waveguide mode is excited at $\Gamma_{\text {inc }}$ with an analytically given amplitude profile $\Psi(y)$. The objective is measured at (multiple) point(s) on $\Omega_{\mathrm{E}}$.

$$
\sum_{e=1}^{M}\left(\mathbf{T}^{e} \ddot{\mathbf{u}}+\mathbf{R}^{e} \dot{\mathbf{u}}+\mathbf{S}^{e} \mathbf{u}+\mathbf{g}^{e}-\mathbf{f}^{e}\right)=0
$$

where $\left({ }^{\cdot}\right)=d / d t,\left(^{*}\right)=d^{2} / d t^{2}$, and $M$ denotes the number of elements. We integrate Eq. (2) by a dispersion reducing scheme that can be found in [34]. Herein, the expressions for the element-level constituent system matrices $\mathbf{T}^{e}, \mathbf{R}^{e}, \mathbf{S}^{e}$, $\mathbf{g}^{e}$, and $\mathbf{f}^{e}$ are also derived.

\section{CHARACTERIZING AND QUANTIFYING SLOW-LIGHT BEHAVIOR}

There are different ways to determine if a (PhC) structure for a given geometry or material distribution exhibits slow-light behavior. A common method is to study the dispersion relation $\omega(k)$ from which the group velocity can be computed by

$$
v_{g} \equiv \frac{d \omega}{d k}=\frac{c}{n_{g}},
$$

where $k$ is the wavenumber. In the rightmost expression, the dimensionless group index $n_{g}$ is introduced as an alternative measure for the slow-light behavior, and we can obtain that by numerical differentiation, once $\omega(k)$ is known.

We have performed an eigenvalue analysis to obtain the dispersion characteristics for the supercell structure in Fig. 2(a). In conjunction with the dispersion diagram in the left part of Fig. 2(b), the group index versus normalized frequency plot in Fig. 2(c) reveals that inside the bandgap $a / \lambda=0.21-0.30$, the waveguide mode exhibits (semi)-slowlight behavior with very little group-velocity dispersion at group velocity $v_{g} \approx c /(40 \pm 10 \%)$. The useful bandwidth of the structure (i.e., the range over which the group index remains constant within $\pm 10 \%$ ) is $B_{\mathrm{FWHM}} \approx 12 \mathrm{~nm}$ centered around the normalized frequency $a / \lambda=0.2163$. Consequently, the given supercell structure serves as a suitable starting guess for the optimization process. The geometry has been found via a parameter search in which the radius of the three nearest neighboring holes to the waveguide has been varied [20,44].

For time-domain simulations, it is not that straightforward to retrieve the dispersion characteristics as they appear in the band diagram [45]. Another reliable way to detect slow-light behavior is to analyze the transmission spectrum, which we 


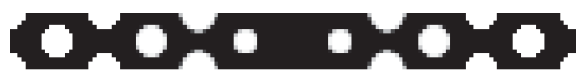

(a)

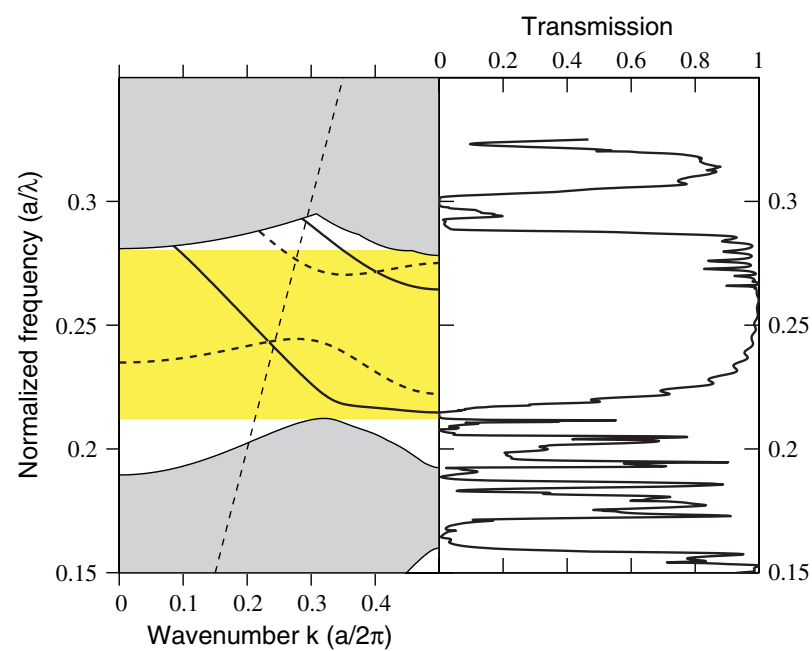

(b)

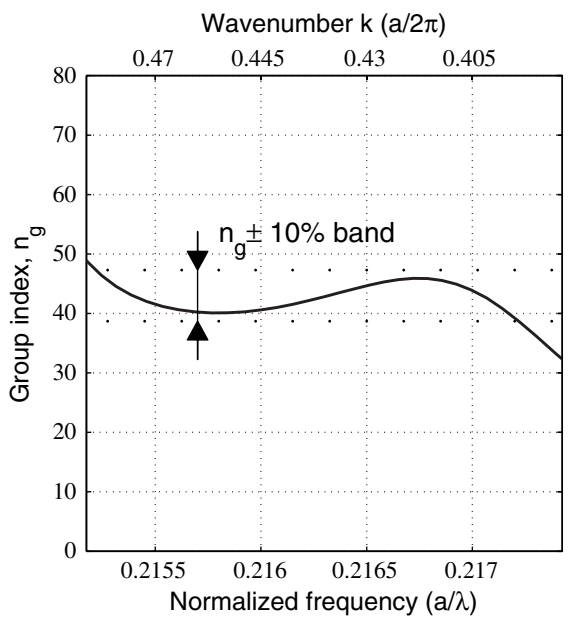

(c)

Fig. 2. (Color online) (a) Rotated supercell used in the eigenvalue computation to obtain the (b) band diagram. This shows the normalized frequencies versus normalized wave vectors for an even (solid) and odd (dashed) PhC-WG mode in the bandgap, and the dashed line indicates the light line above which leaky modes live. The dielectric and the air band correspond to the lower and upper gray dense mode regions, respectively. (c) Group index $n_{g}$ versus normalized frequency.

construct by exciting the inlet ridge waveguide with a short pulse. We obtain the transmission spectrum by integrating the frequency-dependent Poynting flux through a vertical line located after the PhC-WG. Because the PhC-WG sample has a PhC mirror mechanism-the PhC-WG and the nonoptimized in- and outlet-it functions as a so-called Fabry-Perot (F-P) cavity. This leads to F-P fringes in the transmitted energy, and the group velocity can be determined by $[\underline{16}, 17]$

$$
v_{g}=c \frac{2 L_{c} \Delta \lambda}{\lambda^{2}}
$$

where $\Delta \lambda$ is the free spectral range between adjacent F-P peaks, and $L_{c}$ is the length of the (closed) cavity. Matching the transmission spectrum against the band diagram in Fig. 2(b) indicates an unambiguous correlation between the eigenvalue analysis and our finite-element time-domain data. All the characteristics of the dispersion behavior of the PhC-WG mode are reflected in the transmission spectrum. For example, the cutoff for the guided PBG mode at $a / \lambda=0.2136$, the transmission noise due to folding of the upper even mode at $a / \lambda=0.2627$, and the gradual decrease of the spectral distance $(\Delta \lambda)$ between the $\mathrm{F}-\mathrm{P}$ fringes in the frequency range $a / \lambda=0.21-0.25$ toward the cutoff [insinuating slow-light behavior-see Eq. (4)] are all distinctively resembled in the transmission spectrum. Further, apart from the energy transmission, Fig. 3 shows the reflected energy recorded at a vertical line in front of the PhC-WG, from which the energy balance is computed. We clearly see that for the wavelength range corresponding to the linear part of the guided dispersion curve, all input energy is transmitted through the PhCWG, automatically yielding almost-zero reflection. Hence, very little coupling losses between the ridge and the $\mathrm{PhC}$ WG occur. The energy balance is almost conserved here due to high mode confinement in the PhC-WG. Around the bandgap edges, the energy balance is disrupted partly due to poor coupling at the $\mathrm{PhC}-\mathrm{WG}$ entrance resulting in reflected and surface modes not captured by the vertical line in front of the PhC-WG. The poor mode confinement in the slow-light region can give rise to a lateral mode flow, which is not included in the computed energy flow and therefore further explains why the energy is not preserved.

Where the above techniques require frequency information, the time-of-flight method (see, e.g., $[46,47]$ ) relies on time observations of the pulse delay $\eta$ as it traverses the PhC-WG to compute the group velocity by
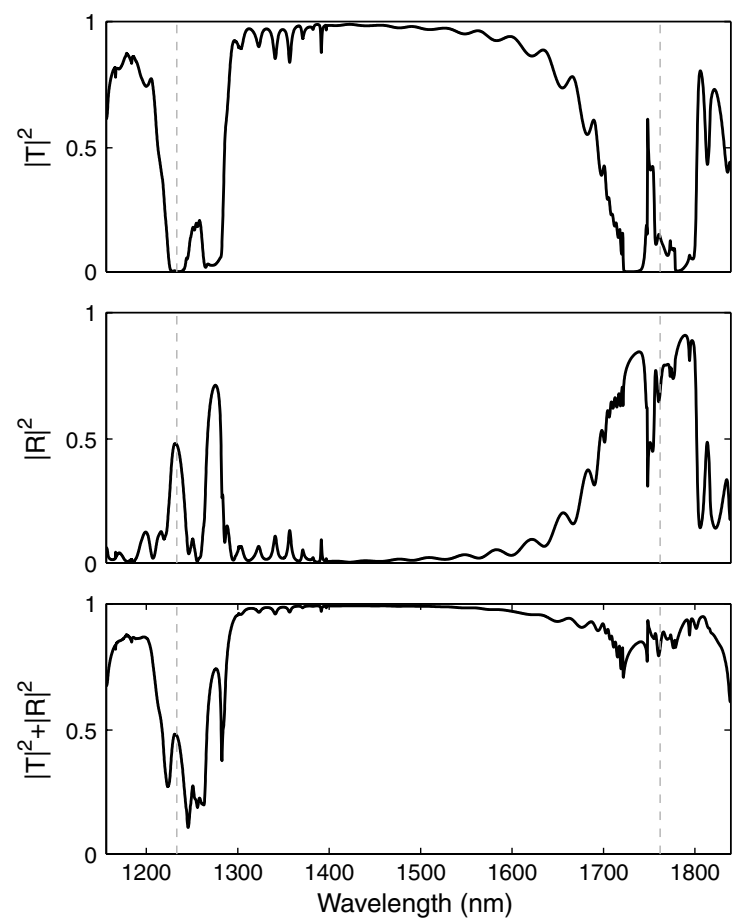

Fig. 3. Transmitted- $|T|^{2}$, reflected energy $|R|^{2}$, and energy balance $|T|^{2}+|R|^{2}$ through in- and outlets as a function of the wavelength for the initial solution (and thus the sum of the reflection and transmission does not equal 1). The interval between the dashed lines indicates the bandgap region. The crystal lattice is assumed to be $a=370 \mathrm{~nm}$. 


$$
v_{g}=\frac{\eta}{L}
$$

where $L$ is the length of the PhC-WG. For the device in Fig. $\underline{1}$, the time delay $\eta$ can be determined by recording the probe pulse at a given point in the exit ridge waveguide with and without the PhC-WG. For a precise measure, we extract the envelope of the probe pulses and define the delay as the temporal distance between the envelope peaks. It is important to be sufficiently downstream in the ridge waveguide to avoid potential near-field disturbances from surface modes living along the in-/outlet interface.

The definition in Eq. (5) gives us a quantitative measure, namely the delay $\eta$, which can be adopted in the formulation of a time-domain optimization problem.

\section{OPTIMIZATION PROBLEM}

The optimization problem with the setup given in Fig. 1 is based on pulse shaping of the envelope $r(t)$ of the probe signal $u(t)$, registered at $\Omega_{\mathrm{E}}$ in the ridge waveguide. The aim is to distribute air and dielectric material in the design domain $\Omega_{\mathrm{D}}$, such that the envelope signal obtained at (multiple) registration point(s) is delayed as much as possible while following a prescribed envelope, obtained from the same input signals propagating in a straight ridge waveguide. The pulse conforming measure is defined as [33]

$$
f=\frac{\int_{0}^{T} \sum_{i \in \Omega_{\mathrm{E}}}\left[r_{i}(t)-\alpha p_{i}(t-\eta)\right]^{2} \mathrm{~d} t}{\int_{0}^{T} \sum_{i \in \Omega_{\mathrm{E}}} \alpha^{2} p_{i}(t)^{2} \mathrm{~d} t},
$$

where $r_{i}(t)$ denotes the envelope of the probe signal $u_{i}(t)$ registered at the $i$ th point in $\Omega_{\mathrm{E}}, p_{i}(t)$ is the prescribed envelope function delayed $\eta$ and scaled by $\alpha$, and $T$ is the termination time of the transient simulation. To render a physically interpretable measure, we have normalized with respect to the prescribed pulse envelope area(s). The envelope of the probe signal $u_{i}(t)$ is retrieved by utilizing the Hilbert transform [33].

For the design parametrization, each element in the design domain $\Omega_{\mathrm{D}}$ is linked to one (density) variable $\rho_{e}$ that varies continuously between $0 \leq \rho_{e} \leq 1$. By adopting a similar concept to that of the solid isotropic material with a penalization scheme, the design variable serves to interpolate the inverse permittivity linearly between two candidate materials [23,48]

$$
\varepsilon_{r}^{-1}\left(\rho_{e}\right)=\left(1-\rho_{e}\right)\left(\varepsilon_{r}^{\mathrm{I}}\right)^{-1}+\rho_{e}\left(\varepsilon_{r}^{\mathrm{II}}\right)^{-1},
$$

where $(\cdot)^{\mathrm{I}}$ and $(\cdot)^{\mathrm{II}}$ designate air and dielectric material, respectively. All design variables are assembled into the global design vector $\boldsymbol{\rho}=\left(\rho_{1}, \ldots, \rho_{M}\right)^{T}$. The continuous design parametrization in Eq. (7) enables the use of gradient-based optimization algorithm of the globally convergent method of moving asymptotes [49] to find optimized designs.

To formulate a pulse-delaying strategy, we introduce $\eta^{\prime}=\eta / s$ as a design variable with $s$ denoting a scaling factor. The shape of the pulse is controlled by treating the design response measure in Eq. (6) as a constraint. The objective is to maximize the delay, which we formulate as

$$
\begin{aligned}
& \min _{\rho \in \mathbf{R}^{M}, \eta^{\prime} \in \mathbf{R}}-\eta^{\prime} \\
& \text { s.t. : governing Eq. }(2) \\
& g\left(\boldsymbol{\rho}, \eta^{\prime}\right)=f\left(\boldsymbol{\rho}, \eta^{\prime}\right) / g^{*}-1 \leq 0 \\
& 0 \leq \rho_{e} \leq 1, \quad e \in \Omega_{\mathrm{D}} \\
& 0 \leq \eta^{\prime} \leq \eta_{\max }^{\prime},
\end{aligned}
$$

where $0<g^{*} \leq 1$ has been introduced to allow for broadening and distortion of the delayed pulse in Fig. $\underline{4}$ after traversing the PhC-WG. The maximum allowable distortion is obtained for $g^{*}=1$ (i.e., the initial pulse shape has been completely destroyed) and is gradually diminished when $g^{*} \rightarrow 0$ (i.e., the output pulse shape is perfect). The gradients of $g$ with respect to the structurally related design variables are found through the adjoint sensitivity analysis method $[33,34,50]$. The sensitivity of $g$ with respect to the temporally related design variable $\eta^{\prime}$ is given by

$$
\frac{\partial g}{\partial \eta^{\prime}}=\frac{\int_{0}^{T} \sum_{i \in \Omega_{\mathrm{E}}}-2\left[r_{i}(t)-\alpha p_{i}\left(t-s \eta^{\prime}\right)\right] \alpha \frac{\partial p_{i}\left(t-s \eta^{\prime}\right)}{\partial \eta^{\prime}} \mathrm{d} t}{g^{*} \int_{0}^{T} \sum_{i \in \Omega_{\mathrm{E}}} \alpha^{2} p_{i}(t)^{2} \mathrm{~d} t}
$$

where the change of variable $\Pi=t-s \eta^{\prime}$ yields $\partial p_{i} / \partial \eta^{\prime}=$ $-s \partial p_{i} / \partial \Pi$, which we approximate by finite differences. It is important to stress that the delay variable $\eta=s \eta^{\prime}$ is several orders of magnitudes larger compared to the structurally related design variables that vary between 0 and 1 . Hence, the sensitivity expression in Eq. (9) has to be scaled adequately through $s$ in order to gain a well-posed optimization problem. A strategy for choosing the scaling parameter is $0 \leq \eta / s \leq 3$, yielding $\eta_{\max }^{\prime}=3$.

Once a material distribution is obtained for a maximum delay and a given allowable distortion $g^{*}$ and $\alpha$, we will start from the resulting design and use the pulse-shaping response function in Eq. (6) as the objective, now with a fixed $\eta$, to minimize the pulse distortion. The optimization problem is now formulated as

$$
\begin{aligned}
& \min _{\rho \in \mathbf{R}^{M}} f\left(\rho, \eta^{\prime}\right) \\
& \text { s.t. : governing Eq. (2) } \\
& \quad 0 \leq \rho_{e} \leq 1, \quad e \in \Omega_{\mathrm{D}} .
\end{aligned}
$$

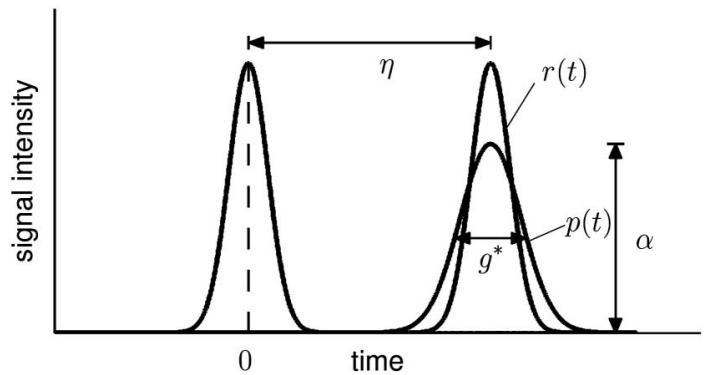

Fig. 4. Pulse delay strategy for pulses with a group delay 0 and $\eta$. The parameter $\eta$ is introduced as a design variable. The $g^{*}$ parameter controls the temporal spreading of the output pulse envelope. The $\alpha$ parameter mainly specifies the transmitted energy, i.e., the amplitude decrease. 
Hence, the optimization is completed in two consecutive steps: (i) a pulse delay step [see Eq. (8)] followed by (ii) a pulse restoring step [see Eq. (10)]. The pulse-shaping constraint in the delay formulation is applied in a single point in the ridge waveguide center sufficiently downstream to allow for larger pulse delays. To achieve perfect transmission for the output signal in the subsequent restoring step, we apply the pulse-shaping objective in multiple points along a vertical line, such that the ridge waveguide mode profile will be inherited automatically in the registered output signal.

\section{MAXIMIZING THE DELAY}

We excite the ridge waveguide by an incident plane-wave Gaussian pulse with a $1 / e$-intensity half-width $T_{0}$ centered around $t_{0}$

$$
\Psi \text { inc }(\mathbf{x}, t)=\Psi(y) \sin \left[k x-\omega_{c}\left(t-t_{0}\right)\right] e^{-\frac{\left(t-t_{0}-x / v_{p}\right)^{2}}{T_{0}^{2}}},
$$

where $\Psi(y)$ is the amplitude profile analytically known for ridge waveguides and $k=\omega_{c} / v_{p}$ is the wavenumber with $\omega_{c}$ denoting the center angular frequency. Further, $v_{p}=c / n$ is the phase velocity with $n=\sqrt{\mu_{r} \varepsilon_{r}}$ designating the refractive index. By utilizing the inverse Fourier transform, the conversion from the full-width at half-maximum (FWHM) bandwidth $B_{\text {FWHM }}$ to the Gaussian $1 / e$ width is obtained by

$$
T_{0}=\frac{\sqrt{4 \ln 2}}{\pi B_{\mathrm{FWHM}}} .
$$

For a specified bandwidth $B_{\mathrm{FWHM}}$, the optimization formulation in Eq. (8) can successfully be deployed to obtain a device, exhibiting slow-light behavior by exciting the structure with the incident wave in Eq. (11) in which $T_{0}$ is determined by Eq. (12). To give the optimization problem design freedom and opportunity for a significant slowdown effect, we choose an operational FWHM bandwidth $B_{\mathrm{FWHM}}=6 \mathrm{~nm}$, and the driving frequency is $\omega_{c} a / 2 \pi c=0.2163$.

To retain a certain periodicity and symmetry of the final design, we only allow for design freedom in the $x$-mirrored part of the in-/outlet region $\Omega_{\mathrm{IO}}$ as well as in the $x-y$ mirrored part of the supercell $\Omega_{\mathrm{SC}}$, distributed periodically downstream over the lattice distance $17 a$ in the design domain $\Omega_{\mathrm{D}}$ (see the symmetry details in Fig. 5). Even though the actual design update is carried out only in a small part of the design domain,

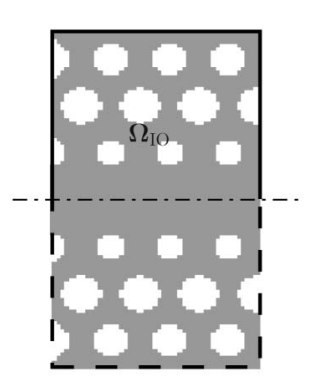

(a)

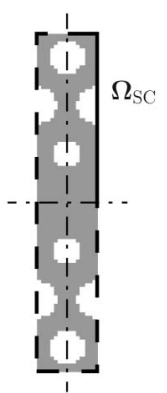

(b)
Fig. 5. Symmetry conditions for the "active" design set. (a) $x$ symmetry is imposed for $\Omega_{\mathrm{IO}}$. (b) $x-y$ symmetry is imposed for $\Omega_{\mathrm{SC}}$. This reduces the number of active design variables further. we still need to compute the sensitivities in the entire design domain, and we subsequently map and sum those properly for the "active" design variables. In Fig. 1, the active design set $\Omega_{\mathrm{IO}} \cup \Omega_{\mathrm{SC}}$ is highlighted in relation to the entire design domain $\Omega_{\mathrm{D}}$. It should be combined with Fig. $\underline{5}$ to clarify the symmetry conditions.

The geometrical restrictions will reduce the optimization design space whereby potentially well-performing designs are disregarded because nothing dictates that a symmetric and periodic structure should be the best performing. However, by limiting the optimization to the in-/outlet region and the supercell, we can directly determine the dispersion characteristics of the resulting structure by a simple eigenvalue analysis of the supercell. The periodicity also enhances manufacturability, that is further boosted by standard density filtering of the design variables with filter radius $R=2.5 \Delta x$, combined with material phase projection [51]. Even though the applied projection function does not preserve the minimum structural length scale for some settings [39], it results in well-defined designs; see Figs. $\underline{6(\mathrm{~b})}-\underline{6(\mathrm{~d})}$ and $\underline{\text { 8(c) }}$. It should be emphasized that whenever an optimized design is presented, we only illustrate the in-/outlet region in conjunction with the supercell structure.

The first part of the optimization has been dedicated to investigate the design as well as the time delay response to three different values for the relaxation parameter $g^{*}=0.001,0.005$, and 0.05 and $\alpha=1$ of the prescribed pulse envelope area in the pulse-shaping constraint. The designs and time delays appear in Fig. 6. For the lowest relaxation factor $g^{*}=0.001$, the pulse is delayed $1.5 \mathrm{ps}$ which doubles to $3 \mathrm{ps}$ for $g^{*}=0.05$. However, the price for the largest delay is a considerable broadening of the pulse and amplitude distortion, which corresponds to an unfavorable reduction of the useful bandwidth of the device. In practice, heavy distortion implies that some of the information carried by the pulse has been lost, and

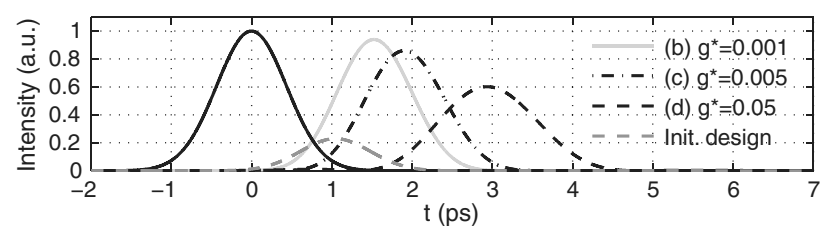

(a)

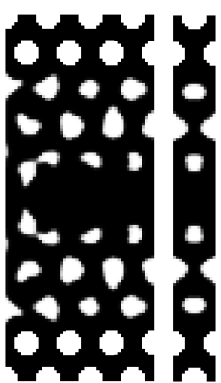

(b)

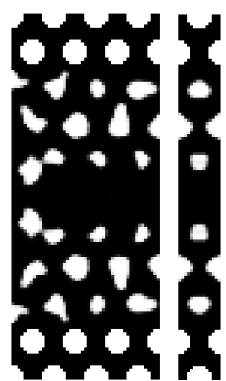

(c)

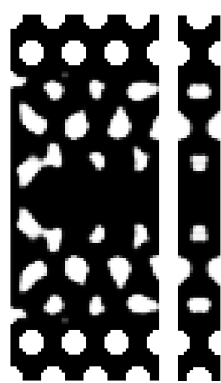

(d) air

dielectric

Fig. 6. Pulse-delayed slow-light designs. (a) Transient intensity response of the ridge waveguide pulse peaking at $t=0 \mathrm{ps}$ and timedelayed pulse for $g^{*}=0.001,0.005$, and 0.05 peaking at $t=1.5 \mathrm{ps}$, $2.0 \mathrm{ps}$, and $3.0 \mathrm{ps}$, respectively. In/-outlet and supercell design for (b) $g^{*}=0.001$, (c) $g^{*}=0.005$, and (d) $g^{*}=0.05$. 
for signal processing devices, it is important to preserve the pulse shape to a certain degree. Further, the peak intensity reduction of a pulse makes it less effective for driving nonlinear effects.

Based on an eigenvalue analysis of the optimized supercell structures the group index versus normalized frequency plot in Fig. 7 confirms that the maximized delays give rise to increased group indices. However, the bandwidth in which the optimized devices can be considered useful for slow-light purposes is simultaneously decreased. The enhanced time delays induce very strong wavelength dependence of the group index. In particular, we see that the "flat-band" region for the initial guess evolves into an S-shaped kink whose center is moving toward the northwest, causing bandwidth shrinkage. For the largest relaxation value, the average group index is $n_{g} \approx 160$, for which, however, the $10 \%$ criterion is not satisfied. This points to the fact that a bandwidth extension is only obtained at the cost of the group index. Frankly, this detrimental effect is inherently associated to slow-light systems, leaving the designer of such systems with a difficult task.

All the optimized designs have reached convergence within 300 optimization iterations. In addition to changing the supercell layout, the geometry of the in-/outlet region has been altered to lower the interface penalty losses, when coupling the access waveguide mode into the PhC-WG. For

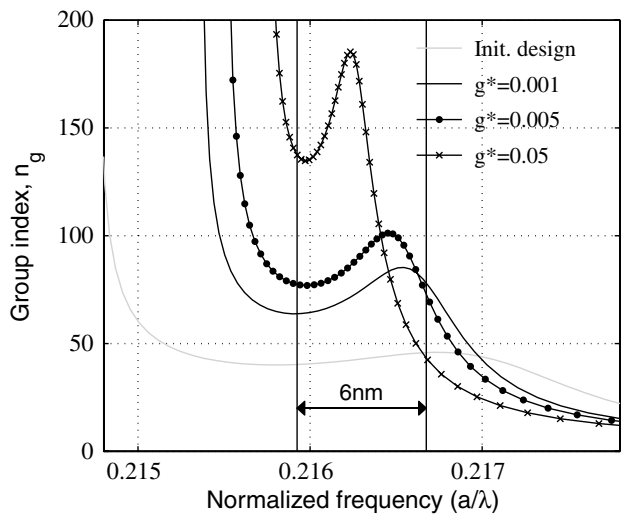

(a)

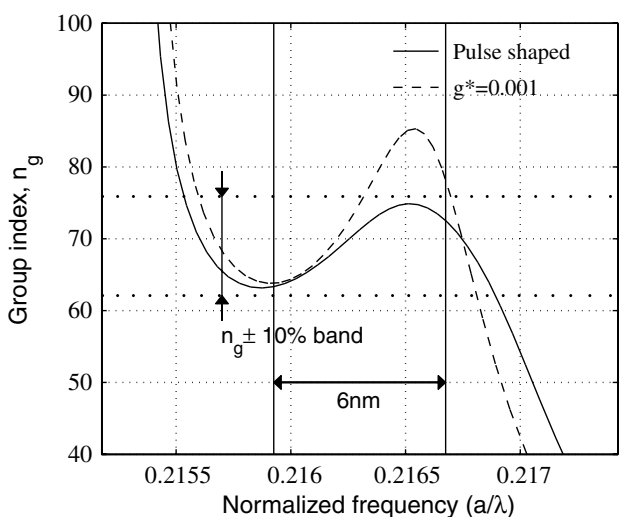

(b)

Fig. 7. Group index versus normalized frequency. (a) Optimized design obtained from the delay formulation in Eq. (8) for three different values for the pulse relaxation: $g^{*}=0.001, \overline{0} .005$, and 0.05 . (b) A comparison between the pulse delayed design for $g^{*}=0.001$ and the pulse delayed-shaped design. real applications, it is important to terminate the $\mathrm{PhC}$ properly, because coupling losses, together with backscattering losses, as a result of fabrication disorders, will degrade the functionality of the slow-light device. Intriguingly, a comparison of the optimized supercell structures in Figs. 6(a)-6(c) and the initial supercell layout in Fig. 2(a) reveals that the optimization has reduced the size of the air inclusions. We consider this a logical action in order to reduce backscattering losses as they originate from the overlap of the optical modes and the hole surfaces [44].

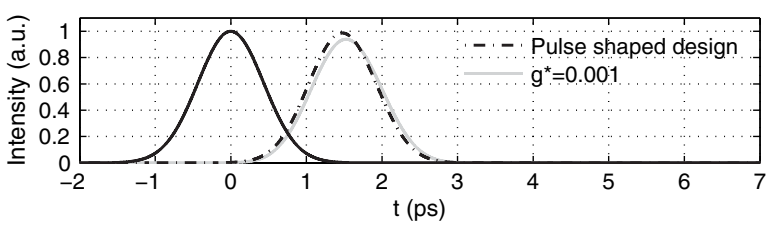

(a)

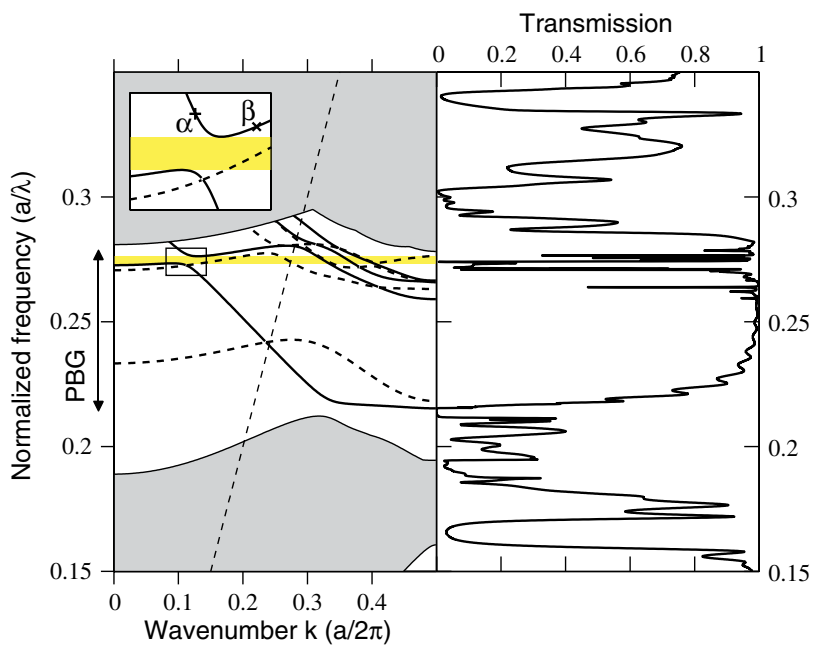

(b)
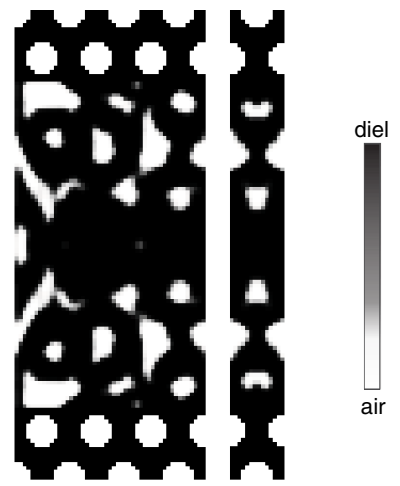

(c)

Fig. 8. (Color online) Pulse-delayed-shaped slow-light design. (a) Intensity response of the initial envelope, peaking at $t=0 \mathrm{ps}$, and the optimized envelope peaking at $t=1.5 \mathrm{ps}$ with and without pulse shaping. (b) Band diagram for the optimized supercell structure and the transmission spectrum for the structure of finite length without optimized in-/outlets. The dashed line is the light line, and the solid and dashed dispersion curves represent the even and odd modes, respectively. The inset magnifies the anticrossing curves in the square with $\alpha$ and $\beta$ indicating the fundamental and higher order modes, respectively. (c) The optimized in-/outlet region (left) and supercell (right). 


\section{RESTORING THE RIDGE WAVEGUIDE PULSE SHAPE}

In Fig. 7(b) it is seen that even for the lowest relaxation factor $g^{*}=0 . \overline{001}$, we obtain a supercell design with a group index variation around $n_{g} \approx 70$ that is not confined to the allowable $\pm 10 \%$ band for the specified $6 \mathrm{~nm}$ bandwidth. To tweak this unsatisfied behavior, we have executed the pulse restoring step with the design in Fig. 6(a) as the initial design and the maximized delay as the fixed time delay in the pulse-shaping objective. Whereas the delay formulation typically reaches convergence within 300 optimization iterations (it naturally depends on the relaxation parameters), the pulse shape formulation has required 1710 iterations for converging to the design in Fig. 8(b). We believe the reason for this rather high number of iterations lies in the fact that the optimization has two concerns; while modification of the supercell structure will result in a change of the waveguide mode, the in-/outlet design needs to be simultaneously adjusted to eliminate coupling losses. To meet and balance both regards, the optimization will then progress in small increments.

As it appears in Fig. 7(b), the optimized design yields a group index variation that satisfies the $\pm 10 \%$ criterion. It is obvious to pose the question why the pulse-shaping optimization strategy does not result in a completely flat-band region. One explanation could be that the optimization has ended up in a local minimum or that a flat-band region is not physically obtainable. We should also remember that as designers, we are competing against the intrinsic dispersion property of the PhC-WG, that the group index can only be increased at the cost of the operational bandwidth. It is also noted in Fig. 7(b) that the group index versus normalized frequency curve is shifted slightly toward lower frequencies compared to the start design. Based on a comparison of the supercell designs
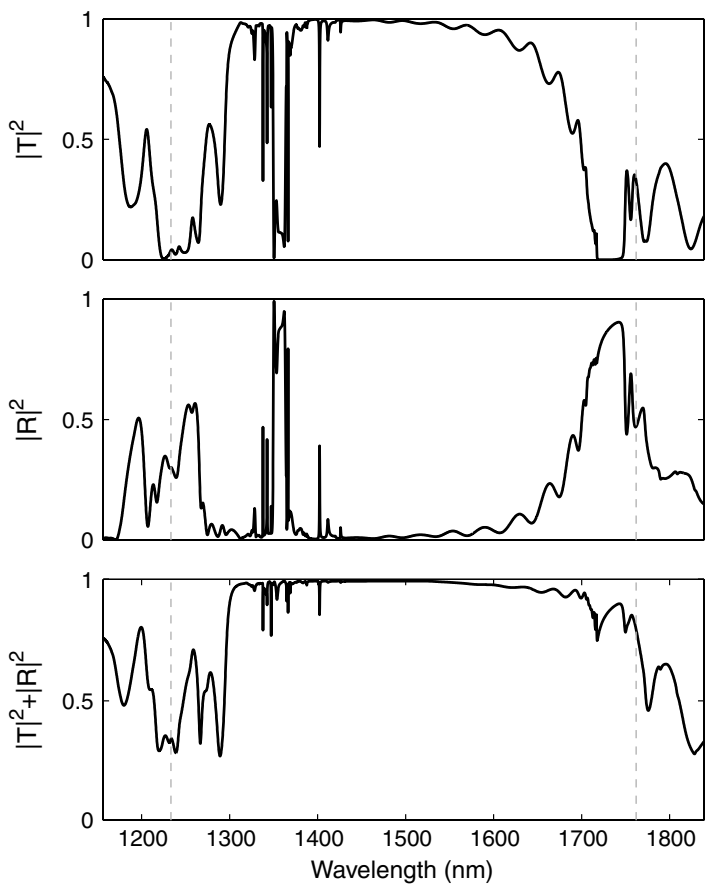

Fig. 9. Optimized supercell design without in-/outlet design. Transmitted- $|T|^{2}$, reflected energy $|R|^{2}$, and energy balance $|T|^{2}+|R|^{2}$ as a function of the wavelength. The interval between the dashed lines indicates the bandgap region. in Figs. $\underline{2(a)}$ and $\underline{8(c)}$ showing that the amount of dielectric material has increased moderately, the horizontal shift of the group index curve is expected, because the frequency of the guided mode scales by $1 / \sqrt{\varepsilon}$ in a medium of dielectric constant $\varepsilon$ [39].

The transmission, reflection, and energy balance spectrum for the finite waveguide structure assembled from the supercell design in Fig. 8(c) without the optimized in-/outlet region is presented in Fig. 9. It shows clearly defined F-P fringes with decreased spacing toward the cutoff at $a / \lambda=0.2153$ in the slow-light wavelength region. Here (i.e., near the band edge), the wavelength spacing is measured to $\Delta \lambda=3 \mathrm{~nm}$, and for an effective $\mathrm{F}-\mathrm{P}$ cavity length $L_{c}=19 a$ this yields $v_{g} \approx c / 70$. The spectrum also contains a dip at $\lambda=1351 \mathrm{~nm}(a / \lambda=0.2739)$ with zero transmission and complete reflection. This spectral behavior is caused by anticrossing of a forward-propagating fundamental mode and a backward-propagating higher order mode pulled down from the air band, giving rise to a mini stopband [52], see Fig. 8(b). These modes live in the upper (lower) part of the design domain due to the increase of dielectric material here.

If we include the optimized in-/outlet design, Fig. 10 shows that almost-perfect transmission, i.e., $|T|^{2} \approx 0.98$, and almost zero reflection are achieved in the wavelength range $B_{\mathrm{FWHM}}=$ $1710 \pm 3 \mathrm{~nm}$ specified by the source. This indicates that no penalty losses are present at the interface due to a mode mismatch, and the associated $H_{z}$ field pattern in Fig. 11(a) for the dominant source wavelength also confirms perfect transmission compared to the poorly performing start design with a field pattern given in Fig. 11(b).

The use of a $2 \mathrm{D} \mathrm{PhC}$ of infinite height in this paper omits the potential influence of the out-of-plane energy losses. However, in the case of slow-light devices, based upon a
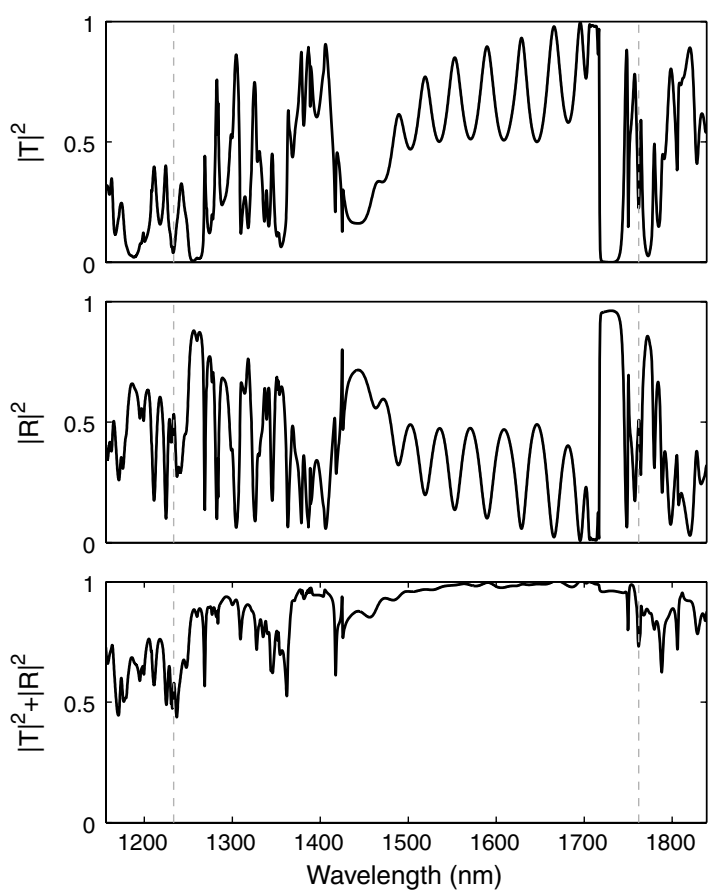

Fig. 10. In-/outlet and supercell design in Fig. 11(a). Transmitted $-|T|^{2}$, reflected- $|R|^{2}$, and energy balance $|T|^{2}+\frac{|R|^{2}}{}$ as a function of the wavelength. The interval between the dashed lines indicates the bandgap region. 


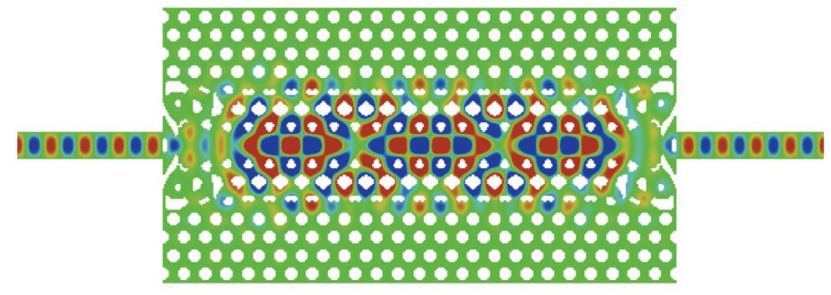

(a)

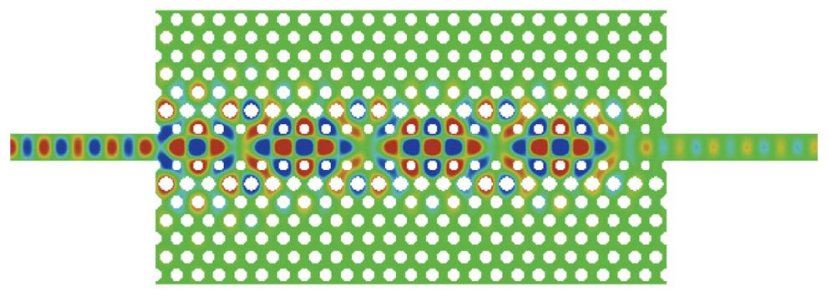

(b)

neg pos

Fig. 11. (Color online) $H_{z}$ field pattern for slow-light device. (a) Optimized structure at $a / \lambda=0.2163$, obtained from delaying and subsequently shaping the pulse. (b) Initial geometry at $a / \lambda=0.2163$. The material distribution is shown with a 0.6 threshold.

W1 PhC-WG, it has been argued that 2D and 3D studies will result in the same qualitative behavior; the only difference is that the guided band for the $2 \mathrm{D}$ structure needs to be blueshifted, i.e., upshifted [20,53]. Also, our experience from previous studies (see [27-30]) is that 2D optimized designs in general yield good behavior in three dimensions as well. In future studies, we will verify the obtained designs by threedimensional simulations and possibly extend the optimization to three dimensions as well.

\section{CONCLUSION}

In this study, a topology optimization methodology has been presented to design slow-light structures subjected to transient responses. The slow-light devices are obtained in two consecutive steps: (i) a pulse delaying step followed by (ii) a pulse restoring step. The first step employs an optimization formulation that delays the probe pulse as much as possible, while controlling the allowable pulse distortion through a pulse-shaping constraint. In the second step, a pulse-shaping objective is used for the maximum delay obtained from the previous step to restore the analytically known mode profile of the ridge waveguide and thereby reduce the amount of GVD such that the group index variation satisfies the $10 \%$ rule [44] for the operational bandwidth.

The optimization process starts from a suitable W1 PhC-WG with in- and outlet connections to ridge waveguides, used to feed the system with an incoming pulse-shaped signal and, after traversing the PhC-WG, to monitor the probe signal, respectively. The initial PhC-WG, which has been found through a simple parameter study by varying the radii of the nearest neighboring holes, has group index $n_{g} \approx 40$ with very low GVD.

To retain periodicity and symmetry in the final designs the active design set is limited to the in-/outlet region as well as to the supercell structure inside the entire design domain. The manufacturability is further enhanced by standard density filtering techniques combined with material phase projection. With these settings, the pulse delaying optimization step yields a structure with group index variation around $n_{g} \approx 70$ (i.e., a $75 \%$ increase) that does not meet the $10 \%$ criterion. However, the subsequent pulse restoring step fixes this issue, and the resulting pulse shape is very close to the equivalent one monitored in a straight ridge waveguide.

The energy flow inside the structures of finite length and an eigenvalue analysis of the supercell confirm that the initial as well as the optimized structures hold the expected slow-light properties.

\section{ACKNOWLEDGMENTS}

This work is supported from the Eurohorcs/ESF European Young Investigator Award [54] through the grant "Synthesis and topology optimization of optomechanical systems," as well as the M.B. Richters Foundation and the Danish Center for Scientific Computing are gratefully acknowledged. The authors are grateful to members of the TopOpt group [55] for fruitful discussions related to the presented work.

\section{REFERENCES}

1. G. Diener, "Superluminal group velocities and information transfer," Phys. Lett. A 223, 327-331 (1996).

2. L. V. Hau, S. E. Harris, Z. Dutton, and C. H. Behroozi, "Light speed reduction to 17 metres per second in an ultracold atomic gas,” Nature 397, 594-598 (1999).

3. M. M. Kash, V. A. Sautenkov, A. S. Zibrov, L. Hollberg, G. R. Welch, M. D. Lukin, Y. Rostovtsev, E. S. Fry, and M. O. Scully, "Ultraslow group velocity and enhanced nonlinear optical effects in a coherently driven hot atomic gas," Phys. Rev. Lett. 82, 5229-5232 (1999).

4. T. Baba, "Slow light in photonic crystals," Nat. Photon. 2, 465-473 (2008).

5. T. J. Karle, Y. J. Chai, C. N. Morgan, I. H. White, and T. F. Krauss, "Observation of pulse compression in photonic crystal coupled cavity waveguides,” J. Lightwave Technol. 22 , 514-519 (2004).

6. R. S. Tucker, P. C. Ku, and C. J. Chang-Hasnain, "Slow-light optical buffers: capabilities and fundamental limitations," J. Lightwave Technol. 23, 4046-4066 (2005).

7. B. J. Eggleton, C. M. de Sterke, and R. E. Slusher, "Bragg solitons in the nonlinear Schrödinger limit: experiment and theory," J. Opt. Soc. Am. B 16, 587-599 (1999).

8. M. Soljacic, S. G. Johnson, S. H. Fan, M. Ibanescu, E. Ippen, and J. D. Joannopoulos, "Photonic-crystal slow-light enhancement of nonlinear phase sensitivity," J. Opt. Soc. Am. B 19, 2052-2059 (2002).

9. M. Soljacic and J. D. Joannopoulos, "Enhancement of nonlinear effects using photonic crystals," Nat. Mater. 3, 211-219 (2004).

10. T. F. Krauss, "Slow light in photonic crystal waveguides," J. Phys. D 40, 2666-2670 (2007).

11. K. Ohtaka, "Energy band of photons and low-energy photon diffraction," Phys. Rev. B 19, 5057-5067 (1979).

12. E. Yablonovitch, "Inhibited spontaneous emission in solid-state physics and electronics,” Phys. Rev. Lett. 58, 2059-2062 (1987)

13. S. John, "Strong localization of photons in certain disordered dielectric superlattices," Phys. Rev. Lett. 58, 2486-2489 (1987).

14. T. F. Krauss, R. M. De La Rue, and S. Brand, "Two-dimensional photonic-bandgap structures operating at near-infrared wavelengths," Nature 383, 699-702 (1996).

15. Y. Akahane, T. Asano, B. S. Song, and S. Noda, "High-Q photonic nanocavity in a two-dimensional photonic crystal," Nature $\mathbf{4 2 5}$, 944-947 (2003).

16. X. Letartre, C. Seassal, C. Grillet, P. Rojo-Romeo, P. Viktorovitch, M. L. dYerville, D. Cassagne, and C. Jouanin, "Group velocity and propagation losses measurement in a single-line photonic-crystal waveguide on Inp membranes," Appl. Phys. Lett. 79, 2312-2314 (2001).

17. M. Notomi, K. Yamada, A. Shinya, J. Takahashi, C. Takahashi, and I. Yokohama, "Extremely large group-velocity dispersion of line-defect waveguides in photonic crystal slabs," Phys. Rev. Lett. 87, 253902 (2001). 
18. H. Gersen, T. J. Karle, R. J. P. Engelen, W. Bogaerts, J. P. Korterik, N. F. van Hulst, T. F. Krauss, and L. Kuipers, "Real-space observation of ultraslow light in photonic crystal waveguides," Phys. Rev. Lett. 94, 073903 (2005).

19. Y. A. Vlasov, M. O’Boyle, H. F. Hamann, and S. J. McNab, "Active control of slow light on a chip with photonic crystal waveguides," Nature 438, 65-69 (2005).

20. L. H. Frandsen, A. V. Lavrinenko, J. Fage-Pedersen, and P. I Borel, "Photonic crystal waveguides with semi-slow light and tailored dispersion properties," 14, 9444-9450 (2006).

21. L. O'Faolain, T. P. White, D. O'Brien, X. Yuan, M. D. Settle, and T. F. Krauss, "Dependence of extrinsic loss on group velocity in photonic crystal waveguides," Opt. Express 15, 13129-13138 (2007).

22. M. Burger, S. J. Osher, and E. Yablonovitch, "Inverse problem techniques for the design of photonic crystals," IEICE Trans. Electron. E87C, 258-265 (2004)

23. M. P. Bendsøe and O. Sigmund, Topology Optimization: Theory, Methods, and Applications, 2nd ed. (Springer Verlag, 2004).

24. J. S. Jensen and O. Sigmund, "Systematic design of photonic crystal structures using topology optimization: low-loss waveguide bends," Appl. Phys. Lett. 84, 2022-2024 (2004).

25. J. S. Jensen and O. Sigmund, "Topology optimization of photonic crystal structures: a high-bandwidth low-loss T-junction waveguide," J. Opt. Soc. Am. B 22, 1191-1198 (2005).

26. W. R. Frei, D. A. Tortorelli, and H. T. Johnson, "Topology optimization of a photonic crystal waveguide termination to maximize directional emission,” Appl. Phys. Lett. 86, 111114 (2005).

27. P. I. Borel, A. Harpoth, L. H. Frandsen, M. Kristensen, P. Shi, J. S. Jensen, and O. Sigmund, "Topology optimization and fabrication of photonic crystal structures," Opt. Express 12, 1996-2001 (2004)

28. L. H. Frandsen, A. Harpoth, P. I. Borel, M. Kristensen, J. S. Jensen, and O. Sigmund, "Broadband photonic crystal waveguide $60^{\circ}$ bend obtained utilizing topology optimization," Opt. Express 12, 5916-5921 (2004).

29. P. I. Borel, L. H. Frandsen, A. Harpoth, M. Kristensen, J. S. Jensen, and O. Sigmund, "Topology optimised broadband photonic crystal Y-splitter," Electron. Lett. 41, 69-71 (2005).

30. J. S. Jensen, O. Sigmund, L. H. Frandsen, P. I. Borel, A. Harpoth, and M. Kristensen, "Topology design and fabrication of an efficient double $90^{\circ}$ photonic crystal waveguide bend," IEEE Photon. Technol. Lett. 17, 1202-1204 (2005).

31. J. Dahl, J. S. Jensen, and O. Sigmund, "Topology optimization for transient wave propagation problems in one dimension: design of filters and pulse modulators," Struct. Multidisc. Optim. 36, 585-595 (2008).

32. L. R. Yang, A. V. Lavrinenko, J. M. Hvam, and O. Sigmund, "Design of one-dimensional optical pulse-shaping filters by time-domain topology optimization," Appl. Phys. Lett. 95 261101 (2009).

33. B. Lazarov, R. Matzen, and Y. Elesin, "Topology optimization of pulse shaping filters using the Hilbert transform envelope extraction," Struct. Multidisc. Optim., published online, doi:10.1007/s00158-011-0642-y (2011).

34. R. Matzen, J. S. Jensen, and O. Sigmund, "Topology optimization for transient response of photonic crystal structures," J. Opt. Soc. Am. B 27, 2040-2050 (2010).
35. J. S. Jensen and O. Sigmund, "Topology optimization for nanophotonics,” Laser Photon. Rev. 5, 308-321 (2011)

36. M. D. Lukin and A. Imamoglu, "Controlling photons using electromagnetically induced transparency,” Nature 413, 273-276 (2001).

37. G. Lenz, B. J. Eggleton, C. K. Madsen, and R. E. Slusher, "Optical delay lines based on optical filters," IEEE J. Quantum. Electron. 37, 525-532 (2001).

38. R. Stainko and O. Sigmund, "Tailoring dispersion properties of photonic crystal waveguides by topology optimization," Waves Random Complex Media 17, 477-489 (2007).

39. F. Wang, J. S. Jensen, and O. Sigmund, "Robust topology optimization of photonic crystal waveguides with tailored dispersion properties," J. Opt. Soc. Am. B 28, 387-397 (2011).

40. O. Sigmund, "Manufacturing tolerant topology optimization," Acta Mech. Sinica 25, 227-239 (2009).

41. F. Wang, B. Lazarov, and O. Sigmund, "On projection methods, convergence and robust formulations in topology optimization," Struct. Multidisc. Optim. 43, 767-784, doi:10.1007/s00158-010 0602-y (2010).

42. J. D. Joannopoulos, S. G. Johnson, J. N. Winn, and R. D. Meade, Photonic Crystals: Molding the Flow of Light (Princeton Univ. Press, 2008).

43. J. Jin and D. J. Riley, Finite Element Analysis of Antennas and Arrays (Wiley \& Sons, 2007).

44. S. A. Schulz, L. O'Faolain, D. M. Beggs, T. P. White, A. Melloni, and T. F. Krauss, "Dispersion engineered slow light in photonic crystals: a comparison,” J. Opt. 12, 104004 (2010).

45. A. F. Oskooi, D. Roundy, M. Ibanescu, P. Bermel, J. D. Joannopoulos, and S. G. Johnson, "MEEP: a flexible freesoftware package for electromagnetic simulations by the FDTD method," Comput. Phys. Commun. 181, 687-702 (2010).

46. K. Inoue, N. Kawai, Y. Sugimoto, N. Carlsson, N. Ikeda, and K. Asakawa, "Observation of small group velocity in twodimensional AlGaAs-based photonic crystal slabs," Phys. Rev. B 65, 121308 (2002).

47. R. S. Jacobsen, A. V. Lavrinenko, L. H. Frandsen, C. Peucheret, B. Zsigri, G. Moulin, J. Fage-Pedersen, and P. I. Borel, "Direct experimental and numerical determination of extremely high group indices in photonic crystal waveguides," Opt. Express 13, 7861-7871 (2005).

48. O. Sigmund and J. S. Jensen, "Systematic design of phononic band-gap materials and structures by topology optimization," Phil. Trans. Roy. Soc. London A 361, 1001-1019 (2003).

49. K. Svanberg, "A class of globally convergent optimization methods based on conservative convex separable approximations," SIAM J. Optim. 12, 555-573 (2002).

50. D. A. Tortorelli and P. Michaleris, "Design sensitivity analysis: overview and review," Inverse Probl. Eng. 1, 71-105 (1994).

51. O. Sigmund, "Morphology-based black and white filters for topology optimization," Struct. Multidisc. Optim. 33, 401-424 (2007).

52. S. Olivier, H. Benisty, C. J. M. Smith, M. Rattier, C. Weisbuch and T. F. Krauss, "Transmission properties of two-dimensional photonic crystal channel waveguides," Opt. Quantum Electron. 34, 171-181 (2002).

53. L. Dai, T. Li, and C. Jiang, "Wideband ultralow high-orderdispersion photonic crystal slow-light waveguide," J. Opt Soc. Am. B 28, 1622-1626 (2011).

54. http://www.esf.org/euriy.

55. http://www.topopt.dtu.dk. 